\title{
A Case of Branchio-oculo-facial Syndrome
}

\author{
Min Young Park, M.D., You Chan Kim, M.D. \\ Department of Dermatology, Ajou University School of Medicine, Suwon, Korea
}

Branchio-oculo-facial syndrome (BOFS) is a rare, autosomal dominant disorder. It is characterized by distinct craniofacial abnormalities including abnormal location of the ears, aplastic cervical skin lesions, malformed auricles, conductive hearing loss, ocular abnormalities, and cleft lip and palate. Herein, we describe a case of BOFS with persistent aplasia cutis of the neck in a 5-year-old girl. (Ann Dermatol 21(3) $288 \sim 290,2009$ )

\section{-Keywords-}

Aplasia cutis, Branchio-oculo-facial syndrome

\section{INTRODUCTION}

Branchio-oculo-facial syndrome (BOFS) is a genetic disorder, first described by Lee et al. ${ }^{1}$ in the early 1980s. It is inherited as an autosomal dominant trait. The clinical manifestations of BOFS range from mild to severe and include eye, ear, oral, and craniofacial abnormalities ${ }^{2}$. Although it is very rare, physicians should be aware of the features of BOFS due to the impact of the associated anomalies. Herein, we describe a case of BOFS with a persistent skin defect of the neck.

\section{CASE REPORT}

In 2003, a 10 day-old girl was admitted to our hospital because of skin defects on the neck under the auricles that had been present at birth. Physical examination revealed aplastic skin with non healing erosions on both sides of the neck, anterior to the sternocleidomastoid muscle (Fig. $1 \mathrm{~A}, \mathrm{~B})$. There was no family history of any similar con-

Received December 12, 2008, Accepted for publication January 8, 2009

Reprint request to: You Chan Kim, M.D., Department of Dermatology, Ajou University School of Medicine, 5, Woncheon-dong, Yeongtonggu, Suwon 443-721, Korea. Tel: 82-31-219-5190, Fax: 82-31-2195189, E-mail: maychan@ajou.ac.kr dition. Other anomalies were noted including a pseudo cleft lip, left branchial sinus, and low set ears with posterior rotation. A normal thymic shadow was identified on the chest X-ray. The abdominal ultrasound showed bilateral hydronephrosis and right uretero-pelvic junction obstruction. There was no evidence of hearing loss, and the ophthalmologic examination showed bilateral nasolacrimal duct obstruction. Chromosome analysis revealed a normal female karyotype. Histological examination of the skin abnormality showed an ulcer with flattened and erosive epidermis, and total absence of the epidermal appendages, which was consistent with a diagnosis of aplasia cutis (Fig. 2A). There were no features suggestive of ectopic dermal thymus such as Hassal's corpuscles on the specimens. The patient was diagnosed with BOFS. The parents declined further evaluation, and the patient was discharged.

At five years of age, the patient visited our dermatology clinic for persistent neck lesions. The skin lesions were more healed than previously, but poorly epithelialized areas remained (Fig. 1C). Skin biopsy specimens showed features of healing skin with flattened epidermis, fibrosis in the dermis and complete absence of adnexal structures (Fig. 2B). The parents reported that surgical repair of the cleft lip and probe insertion of the nasolacrimal duct obstruction were performed at 10 months of age. In addition, during the first year of life, a cyst was found on the posterior scalp, which was removed. The neck lesions were treated conservatively with a simple dressing. We recommended re-evaluation of the eyes, ears and kidneys, and surgical repair for the skin lesions. However, the parents refused further evaluation and invasive treatment. Treatment has included application of topical epidermal growth factor solution and hydrocolloid material dressing.

\section{DISCUSSION}

BOFS is a rare genetic disorder characterized by the presence of distinctive craniofacial, cervical, auricular, 

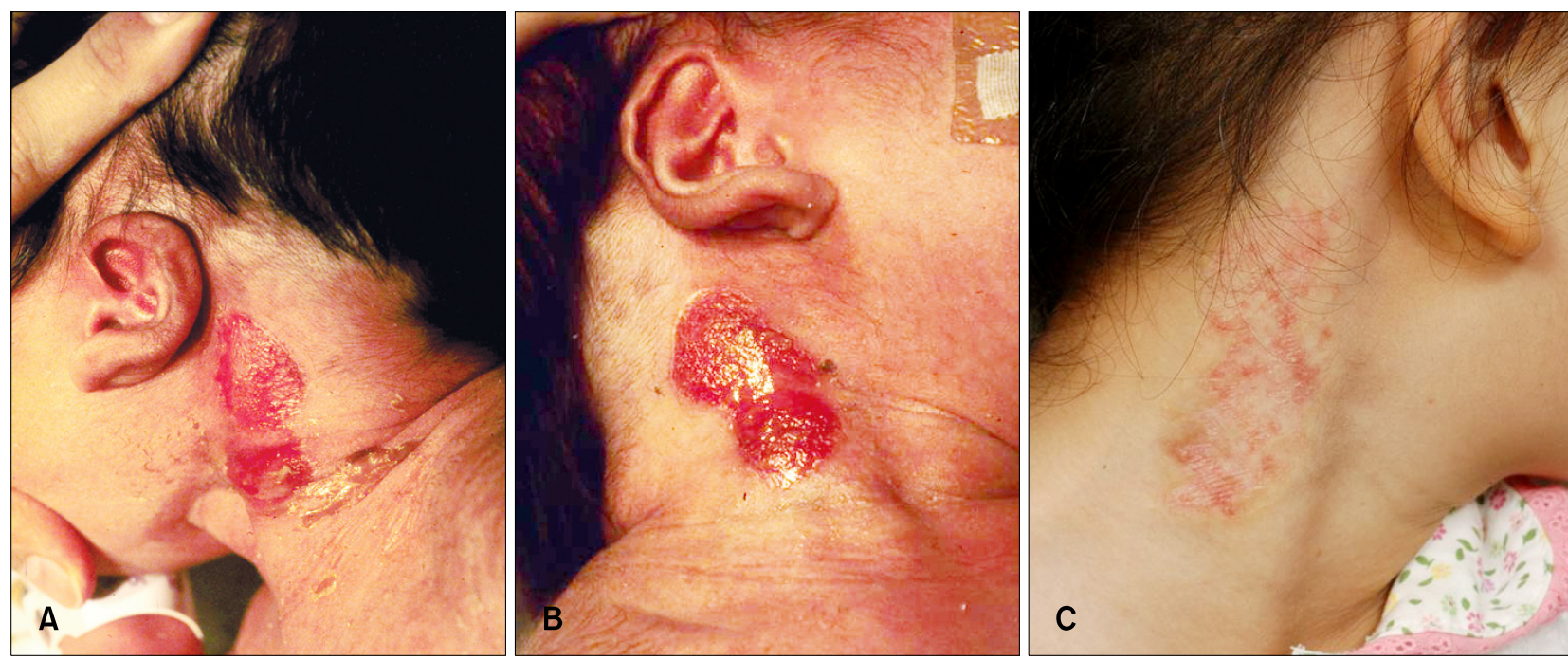

Fig. 1. (A, B) The neonate presented with erosive patches on both sides of the neck. The low set ears with posterior rotation are evident as well. (C) Five years later, the lesion still had poorly healed skin with incomplete epithelialization.
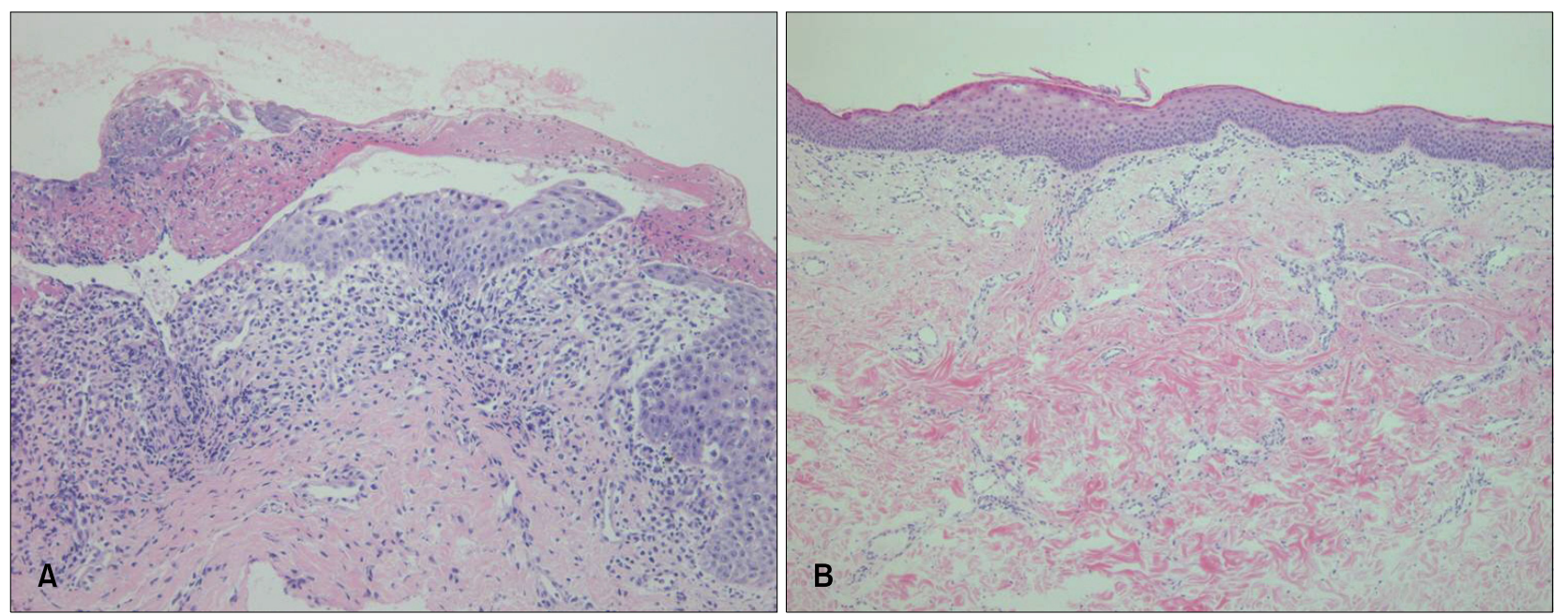

Fig. 2. (A) A skin biopsy from the neck lesion of the neonate showed an ulcer with dermal inflammatory infiltrates and complete absence of appendageal structures. (B) The healing skin demonstrated flattened epidermis with dermal fibrosis. Adnexal structures were not observed $(A, B$ : $H \& E, \times 200)$.

and ophthalmological abnormalities. These may include branchial sinus defects with aplastic skin, low set ears with posterior rotation, cleft lip with or without cleft palate, a pseudocleft of the upper lip, nasolacrimal duct obstruction and microphthalmia ${ }^{2}$. Although the diagnostic criteria have not been clearly defined, the above features when present together usually allow for a clinical diagnosis. In addition, renal anomalies including agenesis, aplasia, hypoplasia, or dysplasia of the kidneys are not infrequent. In some cases, conductive hearing loss, ectopic dermal thymus, scalp cysts, and ectodermal anomalies such as small teeth and dysplastic nails are also noted ${ }^{3}$.
Most of these features were found in our case. BOFS is inherited as an autosomal dominant disorder with incomplete penetrance and variable expression ${ }^{4}$. The chromosomes appear normal, and no gene locus has been identified. However, recently, Milunsky et al. ${ }^{5}$ reported that BOFS is caused by mutations involving the TFAP2A gene, which is one of the AP-2 family of transcription factors. This gene has been shown to regulate the development of facial prominences, limb buds, cranial closure, and the lens vesicle. A genetic study was not conducted in our case because her parents did not agree to have the study done. However, so far the diagnosis of BOFS has been 
based on its clinical manifestations and not on the identification of a mutated gene ${ }^{6}$. Therefore, we consider that a diagnosis of BOFS was reasonable in this case.

The differential diagnosis of the BOFS includes branchiooto-renal syndrome (BORS). Both can be associated with nasolacrimal duct stenosis, hearing loss, and renal anomalies. However, the clinical features such as cleft lip and palate, pseudocleft of the lip, and aplastic skin are not found in BORS. In addition, the responsible genes for the two syndromes appear to be different ${ }^{7}$.

The management of BOFS should be individualized based on the associated anomalies. In some cases, the aplastic skin lesions of BOFS, spontaneously regress leaving a scar-like lesion ${ }^{8}$; in other cases surgical excision and repair are needed ${ }^{9}$. When present, the ectopic dermal thymus may exist on the side with the aplastic lesion; the presence of a normal thymus on chest X-ray should be confirmed before removing the lesion ${ }^{10}$.

In conclusion, we present a rare case of BOFS with a persistent skin defect. Although BOFS is rare, the possible associated anomalies could cause significant problems. A high index of suspicion is needed when a patient presents with clinical features suggesting this diagnosis, to help provide appropriate management and genetic counseling when indicated.

\section{REFERENCES}

1. Lee WK, Root AW, Fenske N. Bilateral branchial cleft sinuses associated with intrauterine and postnatal growth retar- dation, premature aging, and unusual facial appearance: a new syndrome with dominant transmission. Am J Med Genet 1982;11:345-352.

2. Hall BD, deLorimier A, Foster LH. Brief clinical report: a new syndrome of hemangiomatous branchial clefts, lip pseudoclefts, and unusual facial appearance. Am J Med Genet 1983;14:135-138.

3. Lin $A E$, Gorlin RJ, Lurie IW, Brunner HG, van der Burgt I, Naumchik IV, et al. Further delineation of the branchiooculo-facial syndrome. Am J Med Genet 1995;56:42-59.

4. Fujimoto A, Lipson M, Lacro RV, Shinno NW, Boelter WD, Jones $\mathrm{KL}$, et al. New autosomal dominant branchio-oculofacial syndrome. Am J Med Genet 1987;27:943-951.

5. Milunsky JM, Maher TA, Zhao G, Roberts AE, Stalker HJ, Zori RT, et al. TFAP2A mutations result in branchio-oculofacial syndrome. Am J Hum Genet 2008;82:1171-1177.

6. Raveh E, Papsin BC, Forte V. Branchio-oculo-facial syndrome. Int J Pediatr Otorhinolaryngol 2000;53:149-156.

7. Trummer T, Muller D, Schulze A, Vogel W, Just W. Branchio-oculo-facial syndrome and branchio-otic/branchio-otorenal syndromes are distinct entities. J Med Genet 2002;39: 71-73.

8. El Darouti MA, Marzouk SA, Azzam OA, Nada HR, Sobhi RM, El Nabarawi I. Branchio-oculo-facial syndrome with bilateral linear scars of the neck. Int J Dermatol 2005;44: 674-676.

9. Hiroshi F, Satoru S, Eisuke U, Kunihiro K, Yuhei Y. Bilateral dermal thymus of neck in branchio-oculo-facial syndrome. J Plast Reconstr Aesthet Surg 2006;59:1385-1387.

10. Civi I, Kurtay M, Civi S. Bilateral thymus found in association with unilateral cleft lip and palate. Plast Reconstr Surg 1989;83:143-147. 